\title{
INFRAESTRUCTURA DE TRANSPORTE EN EL ASIA-PACÍFICO: DEMANDA FUTURA, PROBLEMAS Y SOLUCIONES
}

\author{
Jonseok Kim \\ InSTITUTO DE TRANSPORTE DE COREA
}

$\mathrm{L}$

a región Asia Pacifico ha emergido como uno de los centros económicos del mundo, y esta tendencia se fortalecerá en el futuro. Para el 2010, el PBI de la región representará más del $30 \%$ del PB1 del mundo y el volumen de tráfico alcanzará casi el $50 \%$ del total mundial. Como consecuencia de esto, la mayoría de instalaciones de transporte, como los puertos marítimos y los aeropuertos, soportan actualmente una severa congestión. Se están estableciendo $e$ implementando nuevos planes de acción, y pronto estarán a punto.

El futuro parece brillante, pero hay, en realidad, muchos obstáculos que superar para hacerlo realidad. La inversión en infraestructura la hace principalmente el Gobierno con su propio presupuesto. La mayor parte de esta infraestructura es de propiedad del Gobierno, que la administra con estrictos reglamentos de operación. Más aún, la competencia internacional entre la infraestructura de transportes se agravará en el futuro cercano. Es probable que parte de la infraestructura que consume tanto dinero se convierta en un fracaso financiero.

En este documento revisaremos el crecimiento económico de la región del Asia Pacifico y su impacto sobre la infraestructura de transporte. Se comparará la capacidad existente con la demanda proyectada y se examinarán los planes de desarrollo de la inversión en la región. Se analizará điversos problemas relacionados con precios, propiedad, administración, financiamiento y regulación de la infraestructura. Se propone posibles soluciones en el contexto de la economía mundial que se está liberalizando.

El crecimiento económico y la infraestructura de la región Asia Pacífico

La región Asia Pacífico ha registrado un rápido crecimiento económico y se proyecta que éste continúe en el futuro. El 
crecimiento anual del PBI de las regiones desarrolladas, como Norteamérica y Europa Occidental, permanecerá en algo más del 2\% hasta el año 2010 y el PBI llegará a más de 9 billones de dólares. Se proyecta que la región Asia Pacífico registre una tasa de crecimiento más alta, algo más de $5 \%$, y que el PBI total de la región llegue a más de 10 billones de đólares, excediendo los totales de Norteamérica y de Europa Occidental. La participación del PBI total de la región en el total mundial llegará a $37,1 \%$. Entre los países de la región del Asia Pacífico, se espera que China, Vietnam, Malaysia y Tailandia registren el mayor crecimiento, entre $6 \%$ y $10 \%$. Los países recientemente industrializados (NIC), como Corea, Taiwan, Singapur y Hong Kong, deben registrar una tasa de crecimiento más lenta, de alrededor del $5 \%$. Estas cifras significan, simplemente, que la región se convertirá en un centro económico importante en el mundo.

El rápido crecimiento económico se traduce en un crecimiento veloz del tráfico de pasajeros y carga hacia adentro $y$ hacia afuera de la región. Es bien conocido que la tasa de crecimiento del comercio mundial está sobrepasando la tasa de creci- miento económico. Además, la tasa de crecimiento de los embarques de contenedores excede la tasa de crecimiento del comercio mundial. Se espera que la tasa de crecimiento del comercio alrededor de la región del Asia Pacífico sea mayor de $10 \%$ hasta el 2010.

En cuanto al movimiento de pasajeros, esta región concentrará casi el $50 \%$ del total mundial para el 2010. En 1995, la participación de Asia Pacifico en el total mundial fue de $36,2 \%$. En 1990, el movimiento de pasajeros al interior del Asia tuvo la participación más alta; le siguieron las rutas que unen América con el Nordeste de Asia. El siguiente cuadro muestra las rutas hacia afuera y hacia dentro de la región Asia Pacífico para el 2010.

El rápido crecimiento del transporte de pasajeros genera una fuerte presión sobre las instalaciones de los principales aeropuertos de la región. Esto significa que la mayor parte de los aeropuertos internacionales de la región están congestionados.

El Cuadro 2 muestra la capacidad de los principales aeropuertos de la región y la demanda proyectada.

\section{Cuadro 1 \\ RUTAS DE TRANSPORTE AÉREO}

\begin{tabular}{|l|rrr|}
\hline \multirow{2}{*}{ Renta } & \multicolumn{3}{|c|}{ N de pasajerus reu milloues) } \\
\cline { 2 - 4 } & 1990 & 2000 & 2010 \\
\hline & 8,0 & 18,3 & 38,3 \\
Américas - N. E. de Asia & 1,1 & 2,4 & 5,0 \\
Américas - S. E. de Asia & 1,0 & 1,9 & 3,6 \\
Américas - S. O. del Pacífico & 4,7 & 10,24 & 20,3 \\
Europa - N. E. Asia & 5,1 & 10,56 & 20 \\
Europa - S. E. de Asia/S. O. del Pacífico & 57,5 & 130 & 262 \\
Interior de Asia & & & \\
\end{tabular}




\section{CAPACIDAD Vs DEMANDA EN LOS PRINCIPALES AEROPUERTOS}

\begin{tabular}{|c|c|c|c|}
\hline \multirow{2}{*}{ 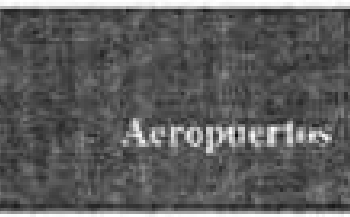 } & \multirow{2}{*}{$\begin{array}{l}\text { Y de Naves de Itinerario } \\
\text { para Pasajeros } \\
\text { Mtovimientos Horas Punti }\end{array}$} & \multicolumn{2}{|c|}{$\begin{array}{l}\text { N de Pasajeros de Itinerario } \\
\text { Plujo/Hurat }\end{array}$} \\
\hline & & Partida & Lesida \\
\hline \multicolumn{4}{|l|}{ Bangkok } \\
\hline 1991 límite declarac & 24 & 3.200 & 4.500 \\
\hline 2000 pronóstico & 48 & 4.650 & 4.680 \\
\hline 2005 pronóstico & 60 & 6.050 & 6.300 \\
\hline \multicolumn{4}{|l|}{ Hong Kong } \\
\hline Capacidad horaria & 32 & 4.600 & 4.600 \\
\hline 2000 pronóstico & 46 & 6.520 & 5.700 \\
\hline 2005 pronóstico & -59 & 7.840 & 7.240 \\
\hline \multicolumn{4}{|l|}{ Seúl-Kimpo } \\
\hline Capacidad horaria & 41 & 3.700 & 3.700 \\
\hline 2000 pronóstico & 57 & 5.150 & 5.330 \\
\hline 2005 pron 6 stico & 74 & 6.820 & 7.250 \\
\hline \multicolumn{4}{|l|}{ Tokio-Narita } \\
\hline Capacidad horaria & 30 & 4.700 & 4.500 \\
\hline 2000 pronóstico & 55 & 9.040 & 9.560 \\
\hline 2005 pronóstico & 69 & 11.690 & 11.530 \\
\hline \multicolumn{4}{|l|}{ Sydney } \\
\hline Capacidad horaria & 60 & 1.500 & 1.500 \\
\hline 2000 pronóstico & 82 & 4.600 & 4.580 \\
\hline 2005 pronóstico & 91 & 6.400 & 6.130 \\
\hline
\end{tabular}

Fuente: IATA (1992) y paises miembros (1994).

En varios lugares, se están planeando e implementando inversiones para la expansión de aeropuertos. Japón comenzó a operar el nuevo aeropuerto Kansai en 1994 y está expandiendo el Narita y el Haneda. Planea construir un nuevo aeropuerto internacional en Chubu, cerca de Nagoya. Corea está construyendo el segundo aeropuerto metropolitano en Incheon, cerca de Seúl. Hong Kong está a punto de abrir el aeropuerto Chep Lap Koken 1998. China, Malaysia, Taiwan y Australia tienen pla- nes de expansión para sus aeropuertos. Sin embargo, a pesar de las inversiones masivas, la capacidad no satisface la demanda esperada para el 2010.

El transporte maritimo muestra una tendencia similar. Debido al rápido incremento del volumen de carga, 24 puertos de la región, incluyendo los de los Estados Unidos, manejaban más de un millón de TEU en 1992. De estos puertos, el de Hong Kong manejaba hasta 8 millones de TEU, 
seguido de cerca por Singapur. Kaoshiung de Taiwan, Pusan de Corea, Kobe de Japón manejaban más de 3 millones de TEU. Tarde o temprano, la capacidad existente de los puertos no satisfará la demanda segúnésta se incremente. El Cuadro 3 muestra la capacidad y la demanda proyectada en varios puertos de la región.

Como se puede observar en los cuadros, muchos de los aeropuertos y puertos marítimos ya están congestionados y no satisfarín la demanda esperada tarde o temprano. Para satisfacer la demanda, los diversos países han establecido planes de expansión y han comenzado a ampliar la infraestructura de transporte. El Cuadro 4 muestra la capacidad existente y los planes de expansión de los principales parses asiáticos con relación a los aeropuertos. Aparte de Estos, Tailandia y Malaysia tienen planes importantes para nuevos acropuertos.

Se planea una inversión similar en la expansión de los puertos marítimos. El Cuadro 5 muestra el resumen de estos planes. Aparte de éstos, Indonesia y Malaysia tienen los suyos propios.

En general, considerando el rápido crecimiento económico y el movimiento del transporte proyectados, las perspectivas económicas para el Asia Pacífico son excelentes. Sin embargo, para adecuarse al crecimiento económico esperado, la expansión de las instalaciones de transporte es esencial. Ésta no es una tarea fácil de lograr con éxito. Los principales proyectos de infraestructura cuestan más de mil millones de dólares; a veces, hasta más de 10 mil millones. El tren de alta velocidad que conecta el corredor entre Seúl y Pusan en Corea cuesta casi 20 mil millones de dólares. El nuevo aeropuerto cercano a
Seúl cuesta más de 5 mil millones de dólares. $¿$ Como es posible financiar tal infraes. tructura con los presupuestos limitados de los gobiernos? Planear una inversión y llevarla a cabo exitosamente son dos asuntos diferentes. Algunos gobiernos son suficientemente ricos para financiar los proyectos de inversión a partir de sus presupuestos. Esto se está volviendo más difícil porque los deseos de la gente se hacen tan sofisticados y diversificados que es casi imposible que el Gobierno los satisfaga. La siguiente sección revisará los problemas que presenta la operación e inversión en infraestructura, suscausas $y$ las posibles formas de resolverlos.

\section{Problemas}

\section{¿Bienes públicos?}

Generalmente se considera que la infraestructura de transporte de un país -carreteras, aeropuertos, puertos marítimos y represas- es un activo que el Gobierno debe proveer. De hecho el Gobierno financia y opera la mayoría de la infraestructura. ¿Significa esto que así debe ser todo el tiempo? El argumento usual para que el Gobierno lo haga es que la infraestructura es un bien público por naturaleza y, por lo tanto, la provisión por parte de la empresa privada es ineficiente debido al fenómeno de "free riders". Es decir, por definición, una vez que el bien público se provee, los que no pagan por su provisión participarán en su consumo a cambio de nada. Si esto sucede, ninguna entidad privada tiene incentivos para invertir en infracstructura. Por lo tanto, el sector privado solo no puede proveer los servicios socialmente necesarios.

En la mayoría de los casos, sin embar- 


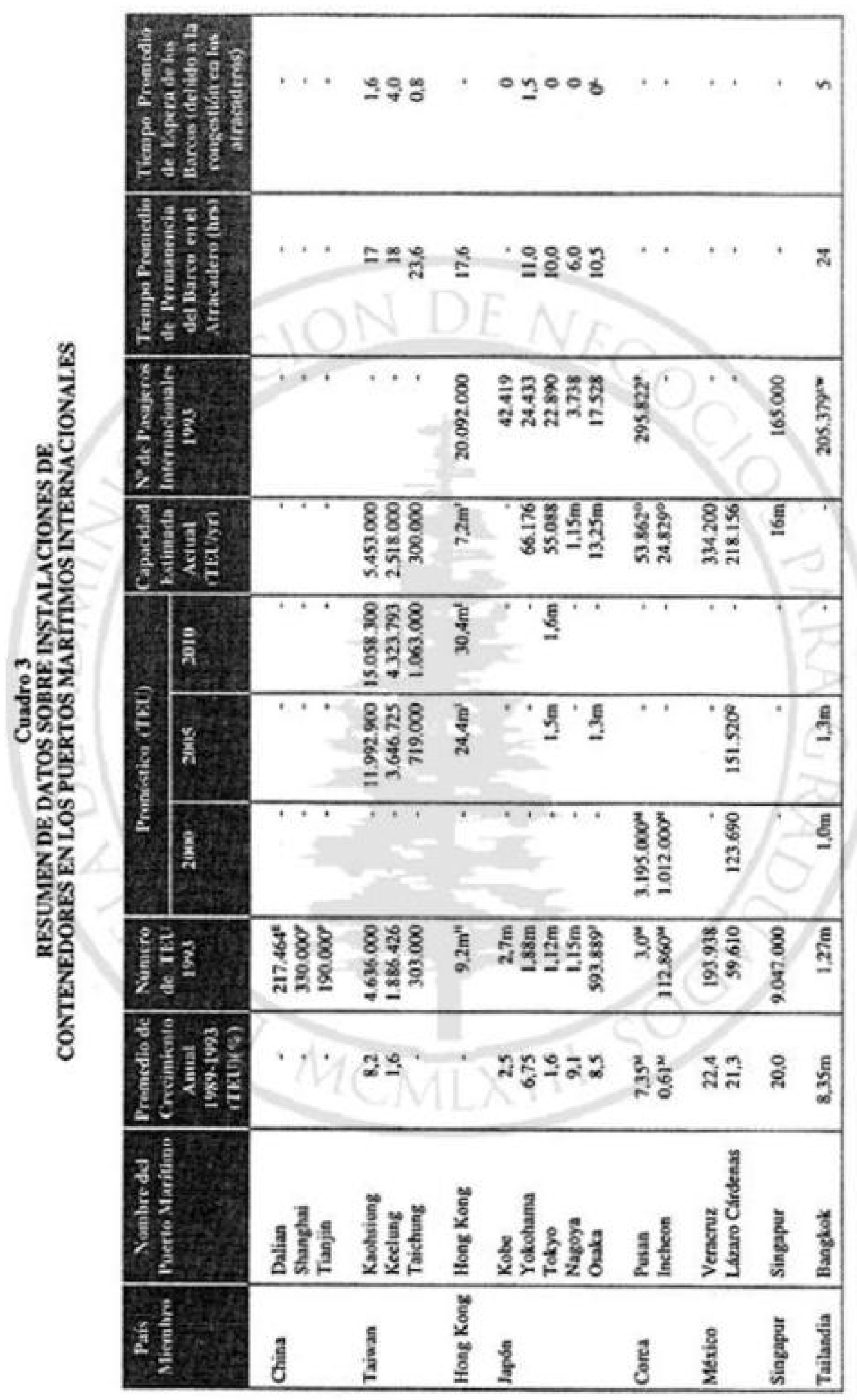




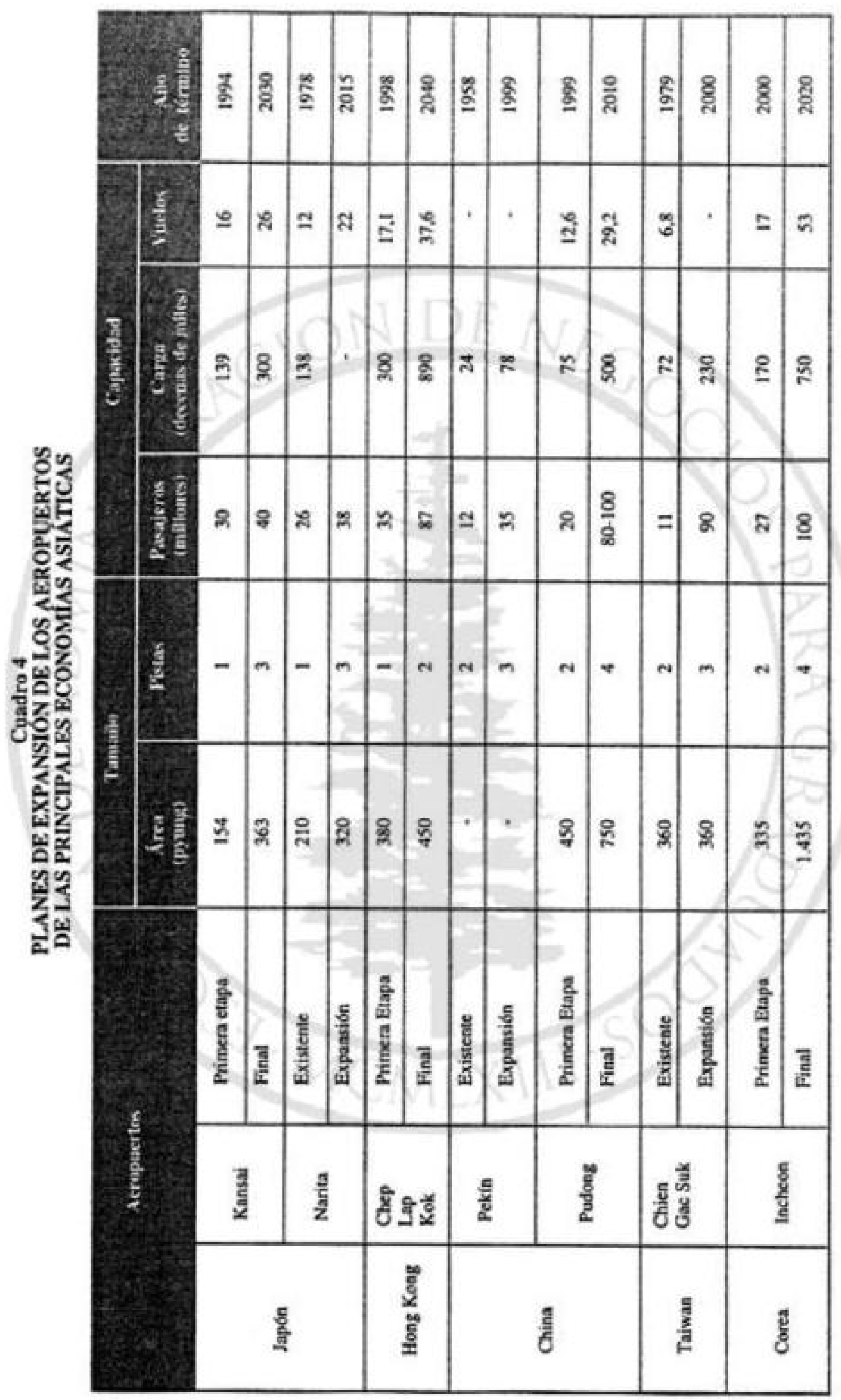




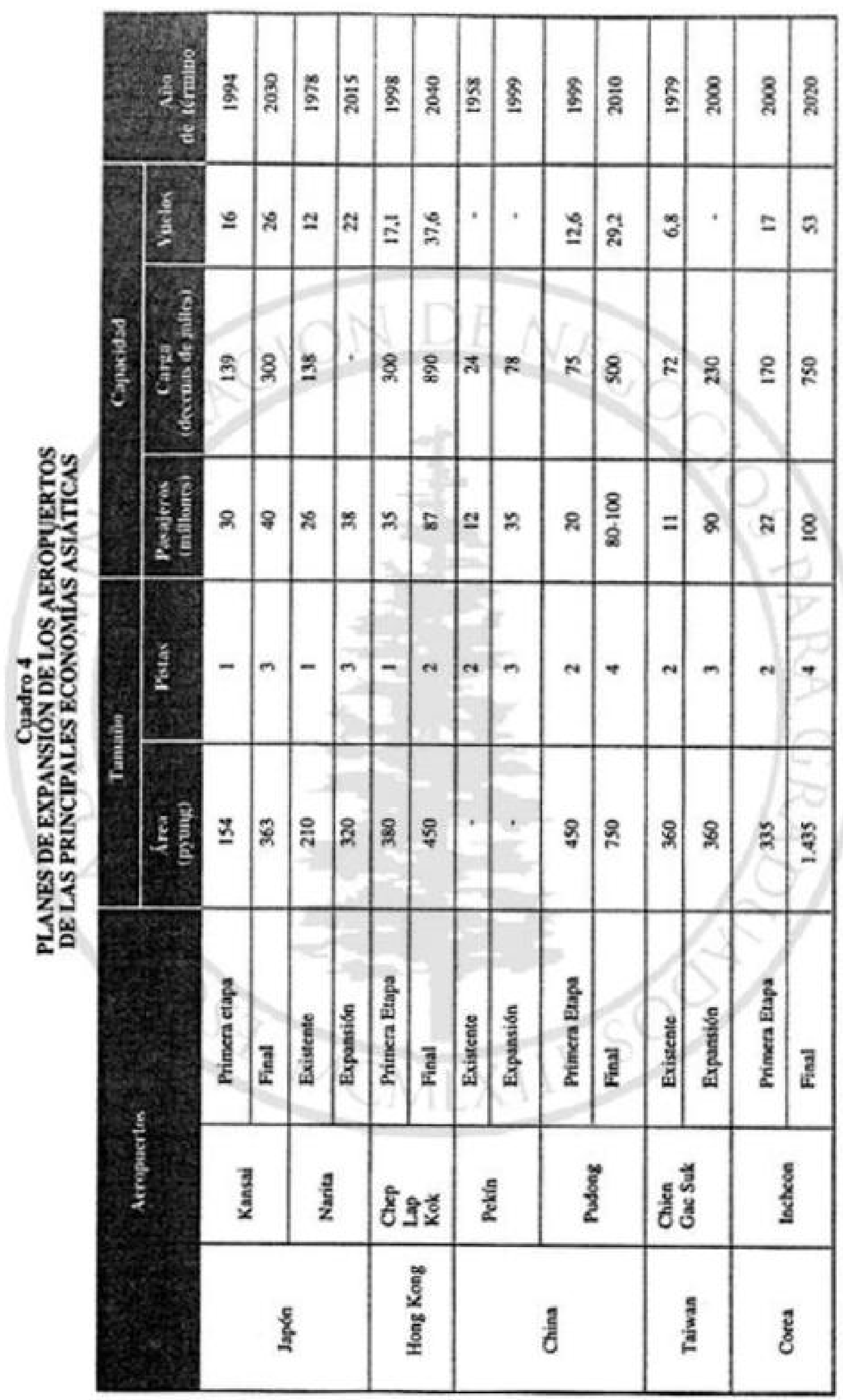


go, lo que la infraestructura provee tiene la característica de un bien colectivo que puede ser consumido por una multitud de personas y, al mismo tiempo, cuyo consumo puede ser controlado y monitoreado a bajo costo por el operador, como un teatro o un avión con muchos asientos. No obstante, se puede argumentar que el Gobicrno debería ser responsable de la inversión $y$ operación de la infraestructura en los casos en que los mercados no estén bien organizados, debido, básicamente, a la infraestructura insuficiente y a la deficiente protección social de los derechos de propiedad individual. Bajo esas circunstancias, el Gobierno es la única entidad que puede organizar y movilizar los escasos recursos de trabajo y capital. Esto significa que el Gobierno debe desempeñar un papel catalizador para la integración y el desarrollo económicos creando la infraestructura física y mental necesaria para la sociedad, como la educación, las carreteras, la electricidad, etc. En la fase inicial de la formación social y el desarrollo económico, este argumento funciona y la mayoría de países siguen este sendero hacia el desarrollo económico.

La validez de este argumento no significa necesariamente que el Gobierno debería ser el único agente en proveer tales servicios. Si los varios mercados se crean y desarrollan hasta cierto grado, entonces el sector privado puede hacerse cargo del papel de proveedor de infraestructura. Esto no significa que no se le deje al Gobierno papel alguno que desempeñar. Debe comprometerse a proporeionar los servicios esenciales a los pobres y a los socialmente débiles en general. También debe ser responsable de manejar las externalidades generadas en la provisión de servicios de infraestructura, tales como el ruido y la contaminación. También debería ser responsable de monitorear el desempeño en términos de la calidad de servicio y el nivel de precio para que el sector privado no ejerza un poder monopólico.

\section{Precios}

El desarrollo de infraestructura en la re. gión del Asia Pacífico no será fácil sin recursos financieros que respalden los procesos de expansión. La manera convencional de conseguir esos recursos es usando los ingresos por impuestos. Este método es ineficiente en el sentido de que los no usuarios de los servicios de infraes. tructura están subsidiando a los usuarios que, de otro modo, pagarían tarifas de usuario más altas o no tendrían servicios en absoluto. Los usuarios normalmente no pagan todo el precio que permitiría al inversionista $y$ al operador recuperar todo el costo de la inversión y de la operación de la infraestructura. Esto significa que el precio bajo induce al exceso de dernanda. Si la intensidad de uso no es excesiva con relación a la inversión, significa simplemente que hay demasiada inversión (en el caso en que la infraestructura sea divisible de alguna manera) o que el proyecto de inversión mismo es económicamente inviable (en el caso en que la infraestructura sea indivisible o divisible). En estos casos, los precios no están funcionando como herramienta para la asignación de recursos. Además, el ingreso así obtenido no cubre los costos de inversión. De esta manera, el financiamiento basado en el ingreso que se espera obtener por la provisión de los servicios de infraestructura no es factible para los inversionistas. La fijación de precios apropiados por los servicios de infraestructura es muy importante para facilitar el financiamiento de los proyectos de expansión. No sólo eso. 
los precios apropiados proporcionarán una señal correcta para la escala de inversión.

\section{Administración deficiente}

La administración eficiente de la infraestructura está también estrechamente relacionada con la facilidad de la financiación de los fondos de inversión necesarios. En términos generales, la mayoría de la infraestructura en la región se maneja ineficientemente, lo que trae como consecuencia una ineficiencia técnica, es decir, no se emplea el método menos costoso para la producción de los servicios. La administración deficiente también provoca el fracaso en la expansión de los mercados y de las fuentes de ingresos valiosas. El resultado combinado de la mala administración es no sólo un aumento del costo, sino una disminución de la demanda y, por lo tanto, del ingreso.

Son dos las causas principales de una administración deficiente: la propiedad de la infraestructura y la regulación de su administración y operación. La infraestructura de la región, excepto la de las economías avanzadas, es de propiedad de los respectivos gobiernos, y también es supervisada por éstos. Por lo general, los gobiernos nombran a la alta gerencia de la infraestructura. La duración en el cargo no depende del desempeño financiero de la administracion, sino de los favores que el Gobierno conceda. En esta situación, los objetivos de la administración se dirigen a buscar el favor gubernamental y no la innovación gerencial y el ejercicio de la capacidad empresarial. Las pérdidas financieras que la infraestructura experimentará serán compensadas con los subsidios del Gobierno.
Los resultados son un mal desempeño financiero $y$ un deficiente nivel de servicio. No se concede incentivo alguno a la innovación técnica en la prestación de servicios. Los déficit se acumularán en detrimento de una administración sana de los presupuestos gubernamentales. La presión inflacionaria prevalecerá debido al déficit presupuestal. Se impedirá la estabilidad macroeconómica.

La regulación de las operaciones también contribuye a la administración deficiente de la infraestructura. La diversificación de servicios es limitada debido a los pocos incentivos de la gerencia para llevarla a cabo. La diversificación del servicio ocurre cuando el proveedor del servicio trata de identificar qué quiere el consumidor y se lo proporciona. La capacidad productiva de la infraestructura se reduce a causa de la restricción de las operaciones, en el caso de los aeropuertos y puertos maritimos. Por ejemplo, la prioridad de maniobra dada a operadores especificos reducirá la capacidad del puerto cuando éste se encuentre congestionado. Si existen atracaderos vacantes, los barcos de los demás operadores deben esperar hasta que se haya terminado con los barcos del operador en cuestión. La restricción de los horarios de trabajo de los camiones que llevarán la carga de los barcos al interior del país causará un atraso en el manejo de los barcos que ya han llegado a puerto. Un toque de queda es otro ejemplo de restricción operativa que constriñe innecesariamente la infraestructura.

\section{Desorden financiero}

Salvo por algunas instalaciones de infraestructura que encaran demandas enormes y crecientes, como la electricidad, la mayo- 
ría de instalaciones de infraestructura sufre de déficit financieros. Por supuesto, no se constituyen fondos para la expansión ni para nuevas inversiones. Como ya se mencionó, varias razones están detrás de esto. La primera de ellas es la distorsión de precios. La segunda es que las restricciones a las operaciones, tales como las obligaciones de servicios públicos, significan una gran carga para el desempeño operativo total de la infraestructura. En este caso, los superávit que se obtenga de algunos tipos de servicio se agotarín debido a una provisión no económica de la obligación de servicios públicos, que perjudica un negocio que, de otro modo, sería rentable. De este modo, no quedan fondos internos de reserva para inversiones futuras. Tercero, se hace más difícil usar fondos gubernamentales para otorgar subsidios externos para la renovación de la infraestructura o para la inversión en expansión. porque el papel del Gobicrno en la mayoría de las economías se está ampliando, y se vuelve relativamente más difícil persuadir a la gente de que pague más impuestos. Esto significa que el Gobierno debe asignar sus ingresos, relativamente constantes, a una mayor gama de actividades.

\section{Inversión distorsionada}

La baja utilización de las instalaciones existentes debido a varias reglamentaciones y a la operación ineficiente crean la necesidad de expandir la infraestructura antes de lo necesario. Además, las tarifas bajas aumentan la demanda $\mathrm{e}$ inducen a una utilización mayor de las instalaciones existentes. Estos factores se combinan y dan la sef̂al de que se necesita expandir la infraestructura antes de lo verdaderamente necesario. Más aún, los polfticos quieren conseguir apoyo de la población para au- mentar la posibilidad de ser reelegidos. La manera más fácil de conseguir este objetivo es construir o expandir la infraestructura social. Esto también contribuye a distorsionar la inversión en términos de tipo. oportunidad y magnitud.

\section{Coordinación deficiente entre \\ infraestructuras relacionadas}

En el caso de las instalaciones de transporte, los puertos principales y las facilidades de acceso se deben manejar coordinadamente para lograr eficiencia. Si el traslado de carga y pasajeros desde su origen hasta su lugar de destino involucra a más de una instalación y a un operador de transportes se dice que hay una cadena de transporte. En este caso, la capacidad de toda la cadena de transporte se determina por la menor de las capacidades del conjunto de instalaciones. La mejora de las concxiones entre diferentes modos de transporte es crucial para fomentar el transporte intermodal. Sin embargo, usualmente no se coordina las operaciones de los modos de transporte, lo que ocasiona malas conexiones, demoras innecesarias $y$ deficiente utilización de las instalaciones.

Los problemas en este aspecto son que el organismo que gobierna cada instalación y el modo de transporte correspondiente actúan separadamente sin coordinación alguna, y que cada operador es regulado de modo que las fuerzas del mercado operen coordinando naturalmente las diversas actividades. Por ejemplo, el organismo que gobierna un puerto marítimo es diferente del de la carretera o ferrocarril de acceso -de y hacia-al puerto. En el mejor de los casos, los operadores que usan el puerto marítimo olas instalaciones de transporte de acceso están oligopolizados. No 
les interesa encontrar oportunidades de negocios para mejorar la rentabilidad porque están protegidos incluso sin hacerlo.

\section{Ambientes cambiantes}

La región del Asia Pacífico se está liberalizando

El mundo se está liberalizando e integrando económicamente. Se está llevando a cabo diversos procesos de integración y en distintos grados. A la Unión Europea sólo le falta un paso para lograr la integración económica total. El Tratado de Libre Comercio Norteamericano está dando pasos similares hacia la liberalización en esa parte de América. La ASEAN, compuesta de países del Sudeste Asiático, persigue la misma meta. El Pacto Andino tiene el mismo carácter en los países de América del Sur. Además de esta integración regional e independientemente de ella, se persigue la liberalización económica mundial dentro del marco de la OMC. La acción unilateral de los Estados Unidos para liberalizar el comercio es otro factor. La APEC es otro intermediario que involucra la liberalización de la región del Asia Pacífico. Habrá que ver cuántos de estos esfuerzos sobrevivirán. Sin embargo, una cosa es segura, la liberalización económica, cualquiera sea la forma que adopte, tocará en algún grado a todos los países del mundo. Esto también significa que todos los paises deben prepararse para ello.

\section{Implicaciones de la liberalización económica sobre la infraestructura de esta región}

Se puede esperar el siguiente impacto de la liberalización sobre la infraestructura:
- Más competencia entre las instalaciones de infraestructura de diferentes paises.

- La formación de una jerarquía de roles entre la misma infraestructura de diferentes paises.

- Más frecuente participación extranjera en la propiedad y/o administración de la infraestructura básica.

- Desarrollo tecnológico, tal como naves aéreas de alcance más largo y cargueros más rápidos y grandes, así como medios de telecomunicación.

- La oligopolización de las industrias correspondientes y barreras más altas para la entrada a estos sectores.

El primer impacto de la liberalización es sólo un proceso antes de que llegue el segundo impacto mencionado arriba. En el caso del transporte internacional, que traslada pasajeros y carga, se anticipa usualmente que el sistema de núcleo y rayo múltiple internacional será una forma prevaleciente de las estructuras de las rutas. Esto significa que en cada continente unas cuantas ciudades serán centros, y otras serán no-centro, de manera que el transporte intercontinental se basará en ciudades centro de cada continente, desde las que se permitirá el traslado a otras ciudades no-centro como destinos finales. La desregulación del mercado de aerolíneas nacionales de los Estados Unidos y la liberalización del transporte al interior de la Unión Europea muestran este patrón. En el proceso existe mucha competencia entre operadores, interesados o ingresantes, hasta que finalmente se estabiliza. Se comprende entonces la gran competencia como un proceso en equilibrio. Mientras tan- 
to, los sobrevivientes de la competencia construyen una fortaleza alrededor de su castillo de instalaciones de aeropuertos y puertos marítimos. La competencia entre operadores es sólo la imagen en el espejo de la competencia entre ciudades y su infraestructura. Las ciudades de cada continente que sobrevivan serán centros, y aquellas que no lo hagan serán no-centros. En otras palabras, se impone una suerte de jerarquía, de modoque los centros sean núcleos y los no-centros sean rayos. No es necesario decir que el cambio tecnologico en el recorrido y velocidad de los vuelos y en la capacidad de los barcos afectara el destino de cada ciudad, además de los esfuerzos que realice cada país.

Una vez establecidos, los operadores tratarán de proteger su castillo del ataque de los entrantes. Puesto que el transporte internacional involucra instalaciones como puertos marítimos y aeropuertos, y éstos están dominados por unos cuantos operadores, se comprende que la entrada a los mercados no será fácil incluso si no existe restricción legal para ello. Además, la estabilidad de la estructura del sector se asegura por medio de habilidades gerenciales. La estructura organizativa de una compañía gigantesca puede imitar a muchas compañías pequeñas, cada una de un tamaño económico eficiente. La administración descentralizada, como los centros de costo o de utilidades $y$ las muchas polfticas de competencia indirecta dentro de una organización, han tenido éxito en superar los crecientes costos unitarios debidos al tamaño excesivo de una compañfa.

Del mismo modo como se forman jerarquías entre las ciudades, los operadores con base en distintos países se ordenarán de manera jerárquica. Los operadores principales conectarán a las ciudades centro y los otros operadores conectarán a los centros con los no-centros, es decir, estos últimos alimentarán a los operadores principales. Naturalmente, estos operadores pueden ser, en parte, propiedad de inversionistas extranjeros. La propiedad extranjera será uno de los resultados de la liberalización económica.

Para incrementar la capacidad competitiva de su infraestructura, todo los países aceptarán que su administración o propiedad esté en manos de extranjeros, ya sea que lo deseen o no estén de acuerdo con ello. Esto comprenderá no sólo las instalaciones de transporte, sino también otra infraestructura, como la educación, las telecomunicaciones, la electricidad, etc. En otras palabras, no habrá ni una piedra rentable que no cambie una vez que se inicie la liberalización.

\section{Prepararse para la liberalización}

Hasta ahora hemos tratado los problemas vinculados con la administración, propiedad y regulación de la infraestructura y hemos previsto que la liberalización, cualquiera sea la forma que tome, estará pronto con nosotros. Tomando estos dos elementos como dados, discutiremos qué hacer con la infraestructura para que no se nos deje atrís en la carrera.

\section{Comprender que la liberalización es inevitable}

Puede haber tantas configuraciones de la sociedad global como gente hay en el mundo. Por el momento, y en términos generales, la sociedad global se compone de dos grupos antagónicos: capitalismo y so- 
cialismo. El tercer mundo podría escoger un equilibrio entre las dos opciones y organizar sus propias sociedades a voluntad. Desde la caída de la Unión Soviética, no ha habido un poder de contrapeso y la sociedad mundial está, se diría, en desequilibrio. Hoy el mundo está demarcado en bloques regionales, cada uno de los cuales quiere expandir su territorio. La elección que enfrentan los países de desarrollo lento y los países en desarrollo es a qué grupo pertenecer. El mantenerse aislados no parece ser una buena estrategia de supervivencia. Estar aislado en este mundo altamente interconectado no puede ser una táctica ganadora. El desarrollo tecno16 gico no permite que las poblaciones fisicamente aisladas estén realmente aisladas. Las personas están expuestas al mundo de afuera; se les crea deseos que el país no puede satisfacer.

\section{Superar los problemas relativos a la infraestructura}

Para mantener una infraestructura internacionalmente competitiva, especialmente en cuanto a las instalaciones de transporte, hay varias tareas por realizar. En este contexto, hay dos opciones de política: los mecanismos de planificación y los de mercadeo.

El Gobierno puede planear con más cuidado la operación y la administración de la infraestructura para maximizar el bienestar social asociado a su utilización. $\mathrm{La}$ inversión en infraestructura nueva o en expansiones se debe basar en cuidadosos análisis de costo-beneficio. Las tarifas a los usuarios se fijan, lo que genera restricciones al financiamiento del presupuesto gubernamental y se puede traducir en cargas impositivas adicionales a las personas. Además, se toma en cuenta $y$ coordina las tarifas entre instalaciones que compiten entre sí o se complementan. El éxito del enfoque del planeamiento, sin embargo, no depende sólo de la adquisición de la información necesaria sobre las preferencias individuales, las condiciones del costo, etc., $y$ en la habilidad para analizar esta información, sino también de cómo deshacerse de los intereses creados que pueden resultar perjudicados en el juego de la planificación. Éste puede ser un obstáculo infranqueable para el planificador.

Por el contrario, el mecanismo del mercadeo está libre de las restricciones informativas que encara el enfoque de la planificación. Los agentes privados, ubicados cerca de la información exacta y necesaria, deciden cómo administrar y operar la infraestructura, cuándoy cuánto invertir, $y$ cuánto cobrar a los usuarios. A pesar de la ventaja informativa, introducir el meca. nismo del mercado en escena se topará con una fuerte oposición de los intereses creados. Otra desventaja puede ser la concentración del poder del mercado en unas pocas manos, con el abuso que esto puede acarrear.

Cualquiera que sea el enfoque elegido, el obstáculo común es saber cómo hacer para manejar a los grupos con intereses creados. Si se logra superar con éxito esta barrera, se puede tomar cualquiera de las dos direcciones -aunque se puede abogar por el mecanismo del mercado antes que por el de la planificación-.

Para introducir el mecanismo del mercado en la infraestructura, podemos pensar en las siguientes opciones:

- La desregulación. 
- El cambio de la propiedad y/o la forma de organización (incluyendo la privatización).

- Contrato de administración.

La regulación de la operación de la infraestruetura conlleva la libre determinación de las tarifas que se cobra a los usuarios, la inversión y otras reglas de operación y la relación entre la gerencia y los trabajadores. Se debe acompañar la desregulación por incentivos apropiados a la gerencia. Si la estructura de incentivos no cambia, la desregulación misma no funcionara. En este contexto se sugiere a veces la corporatización. Ésta armonizará el objetivo de la gerencia con la desregulación. La infraestructura corporativizada se desempeñará sobre una base comercial con algunas restricciones posibles a los objetivos de la administración. La desregulación combinada con el cambio en la estructura de incentivos que experimenta la gerencia debería proveer servicios socialmente apropiados.

Este nuevo incentivo a la gerencia se puede mantener incluso bajo la propiedad del Gobierno. El Gobierno puede seguir conservando la mayor parte de la infracstructura en forma de capital accionario y desempeñar el papel de accionista mayoritario, supervisor del desempeño de la gerencia. Sin embargo, en la mayoría de casos, el desempeño de las corporaciones públicas cuyo accionista principal es el Gobierno no suele ser tan acertado comose esperaba. Esto puede suceder principalmente porque el Gobierno, o la dependencia particular, no tiene incentivo alguno para hacer que la gerencia haga lo mejor que puede con el fin de lograr un desempen̂o socialmente óptimo. El accionista privado tiene todos los incentivos para hacer que la gerencia haga lo mejor que puede con el fin de obtener las ganancias netas máximas. El incentivo del Gobierno como accionista principal no es igual al de los accionistas privados. Esto significa que la gerencia tiene más libertad para satisfacer sus objetivos personales en vez de servir a los intereses del Gobierno. Incluso bajo propiedad privada, la administración puede perseguir su objetivo personal sin que los propietarios lo supervisen. Esta conducta se agudiza bajo propiedad gubernamental.

La privatización se convierte en una opción seria si se espera que la conducta negligente o el descuido de la gerencia bajo propiedad gubernamental sea mucho mayor que con otra opción. La privatización ayudará a reducir el problema de gerencia de la organización y promoverá una mayor eficiencia de la infraestructura. Sin embargo, presenta sus propios problemas. ¿La maximización de la eficiencia individual coincide realmente con la cficiencia social? Esta preocupación tiene mucho que ver con los numerosos abusos de las compañias privadas que se esconden tras el término genérico de fallas del mercado. Los principales ejemplos de esto son el monopolio, las externalidades y otros aspectos relacionados. En esta situación, cómo privatizar la infraestructura en cuestión se vuelve una pregunta crucial. El asunto gira en torno a cómo mantener la competencia bajo propiedad privada.

Manteniendo intacta la estructura de propiedad de la infraestructura, se puede incrementar la eficiencia gerencial contratando fuera parte o toda la administración. Esto contribuirá a reducir el costo unitario del área o servicio particular que se contrate fuera. Los ejemplos usuales son el mantenimiento, la limpieza, el alcantarillado, 
etc. Mejor aun, el núcleo central del trabajo gerencial, incluyendo el planeamiento estratégico de la inversión, la determinación de precios, los tipos de servicios por proveer, es decir, toda la gerencia, se puede encargar a una compañía de consultores externos o a expertos en administración externos. El exito del contrato de administración depende de la independencia con la que la nueva gerencia pueda desempeñarsu tarea y de cómo obtiene recompensa por su trabajo. El contrato de administración tiene la ventaja que la estructura de la propiedad se puede mantener a pesar de que actúe contra la realización completa del beneficio potencial del contrato de administración.

\section{Los efectos de la reforma}

Se espera que las reformas administrativas, organizativas e institucionales generen efectos como los siguientes:

- Racionalización de costos: eliminación de factores redundantes $\mathrm{e}$ inútiles.

- Determinación de precios de acuerdo con las condiciones de la oferta y la demanda; distintas formas de determinación de precios.

- Reorganización de los servicios provistos: eliminación de servicios no rentables y creación de servicios nuevos y rentables

- Coordinación, basada en el mercado, de servicios interrelacionados que compiten o se complementan: el transporte intermodal se facilitará gracias a la desregulación de la reglamentación operativa y a la prestación concertada de servieios relacionados.
- Acumulación suficiente de fondos de inversión mediante reducción de costos y aumento de ingresos: se alivia en parte los subsidios gubernamentales y los déficit presupuestales.

En el mundo real existen obstáculos para lograr los efectos arriba mencionados. Los grupos del Gobierno con intereses creados, los trabajadores, y la administración anterior son posiblemente los desafios más grandes para la reforma. A causa de ellos no se puede iniciar la reforma misma, e incluso si se pudiera, la operación tropezaría con obstáculos. Se debería hacer esfuerzos serios para convencer o coaccionar a estos agentes.

La competencia insuficiente siempre seguirá siendo un problema, incluso si la implementación de la reforma tiene exito. La compañía dominante en el mercado no tiene mucho interís en hacer un esfuerzo para aumentar el ingreso o reducir el costo $y$ tiende a ser indiferente al entorno cam. biante de los negocios, interno o externo. Puede obtener ganancias satisfactorias sin esfuerzo. Cuando se inicia la reforma es muy importante encontrar formas de mantener la competencia.

\section{El papel del Gobiemo}

El Gobierno desempeña un papel importante no sólo en la ctapa inicial de la reforma, sino en la etapa posterior de imple. mentación. Debe idear formas de limitar la oposición, introducir formas contractuales de manera eficiente en el caso de contratos de administración y monitorear el desempeño de la infraestructura reformada. Debe desarrollar varias medidas para verificar objetivamente el desempeño de la gerencia y mantener una actitud vigilan- 
te para preservar la competencia justa. El preservar la competencia sustituye las medidas visibles del desempeño, como la tasa de retorno, la verificación de la calidad del servicio, la productividad del trabajo. la variación de los costos unitarios, etc. Los indicadores visibles de desempeño pueden subir y bajar por razones que el Gobierno no conoce. Por lo tanto, fijar una regla estricta para medir el desempeño gerencial puede subestimar osobreestimar el verdadero desempeño. En este sentido, la competencia no sólo asegura el mejor esfuerzo por parte de la gerencia, sino que alivia al Gobierno del esfuerzo de evaluar. $\mathrm{Si}$ introducir la competencia directa resulta imposible, entonces el Gobierno debe encontrar la mejor unidad de medida para ayudar a evaluar el desempeño de la gerencia. Incluso en este caso, la competencia indirecta es posible por el criterio de comparación.

Si la reforma muestra un desempeño relativamente acertado, el Gobierno debe comenzar a afinar los detalles de la eficiencia individual y social de la infraestructura. El uso de los servicios de transporte genera molestias sociales tales como el ruido y varios tipos de contaminación. Más aún, la congestión en una infraestructura con restricciones de capacidad no envía a los usuarios un signo correcto de la cantidad óptima de uso. En esta situación, el Gobierno puede desempeñar el papel de realinear el incentivo individual del uso al nivel socialmente apropiado. Una medida de política que los gobiernos pueden adoptar para corregir este desalineamiento es el cobro de impuestos.

También se puede esperar que el Gobierno cumpla un papel de árbitro. Algunos proyectos de expansión no cuajan por la oposición de los vecinos. El Gobiemo debería establecer una regla racional de compensación para éstos.

\section{¿Un núcleo o un rayo?}

En el contexto de la competencia internacional, una ciudad con determinadas instalaciones de transporte o telecomunicaciones puede crecer hasta ser el núcleo de una gran región o puede convertirse en rayo. Sin importar la función de la ciudad en la región, la reforma antes mencionada es esencial para eliminar las ineficiencias e incrementar la competitividad de la infraestructuraen cuestión.Supongamosque hay varias ciudades en una región que pueden proveer servicios similares a los usuarios de la infraestructura. En este caso, la estructura que es relativamente más eficiente y competitiva será preferida por los usuarios internacionales si todas las demás condiciones permanecen iguales.

Esto significa que la infraestructura reformada tiene grandes posibilidades de convertirse en un núcleo o por lo menos en un mininúcleo, dependiendo del volumen de usuarios. El nivel de tarifas por los servicios, la capacidad, las características de la ubicación y las condiciones económicas generales son los principales determinantes de la categoría de núcleo de la infraestructura de una ciudad, especialmente en el mundo liberalizado.

Se podría dar varias recomendaciones además de la reforma administrativa:

- El crecimiento de la economía nacional es esencial para producir suficientes usuarios que vayan desde el origen hasta el destino de la infraestructura en cuestión. Algunas ciudades pueden ser particularmente apropiadas para ser nú- 
cleos de usuarios que meramente pasan por la ciudad. Sin embargo, esto es raro. La mayoría de casos se encuentra respaldada por suficientes usuarios que viajan del origen al destino.

- La mayoría de las infraestructuras que compiten entre sí encararín el mismo desafio de la reforma administrativa para lograr la eficiencia. Por lo tanto, procurar el uso más eficiente posible de la infraes. tructura es esencial para proveer servicios diversos a precios más bajos.

- La infraestructura debe ser manejada por quienes tienen en cuenta el factor internacional. La competencia estratégica entre infraestructuras rivales, con relación a la inversión, los servicios prestados y las tarifas cobradas a los usuarios debe ser bien entendida.

- En el mundo dinámico de cambios rápidos en los patrones de demanda (modelo origen-destino, tipo de bienes transportados) y en la tecnología de vehículos y administración, es importante vincular esos cambios a la ventaja rentable de la infraestructura.

\section{Referencias Bibliograficas}

APEC TWG. Congestion Point Study. Borrador final, volume 1, volume 2, Maunsell, 1995.

CHOI, D.; KIM, J, and FINDLAY, C. "Transport Services Liberalization in APEC". Asia Pacific Economic Review. (En prensa).

FINDLAY,C.;PAREDES-MOLINA,R;KIM, J. and RAGURAMAN. Asia Pacific Air Transport: Challenges and Opportunities, Singapur, SINPECC.
HA, H.G. (ed.). Management Renovation of the Transport Facilities: a Focus on Privatizations. The Korea Transport Institute, 1997.

IATA. Asia-Pacific Air Transport Forecast: 1998-2010. January, 1997.

WORLD BANK. Korea Transport Sector: Resource Mobilization Challenges and Opportunities, 1995. 